\title{
Hot One-Temperature Accretion Flows Around Black Holes
}

\author{
Ann A. Esin, Ramesh Narayan, Eve Ostriker \\ Harvard-Smithsonian Center for Astrophysics, Cambridge, MA 02138 \\ Insu Yi \\ Institute for Advanced Study, Princeton, NJ 08540
}

\begin{abstract}
We describe hot, optically-thin solutions for one-temperature accretion disks around black holes. We include cooling by synchrotron, bremsstrahlung, and Comptonization. Our solutions are thermally and viscously stable, with gas temperatures on the order of $T \sim$ $10^{9}-10^{10.7} \mathrm{~K}$. The thermal stability is a direct result of the inclusion of synchrotron cooling.

The new solution branch is related to the advection-dominated solution for a two-temperature gas described by Narayan \& Yi (1995b). It is present only for mass accretion rates less than some critical $\dot{M}_{\text {crit }}$ which depends on the radius $R$ and viscosity parameter $\alpha$. The solutions are advection-dominated for extremely low values of $\dot{M}$. However, for a range of intermediate accretion rates, the new solutions are both hot $\left(T \sim 10^{10} \mathrm{~K}\right)$ and cooling-dominated. Because of this new feature, one-temperature solutions are significantly more luminous than the corresponding two temperature solutions.

The radial profile of the new solutions is unusual. The inner parts of the flow are coolingdominated and have a disk-like geometry, while the outer parts are fully advection-dominated and nearly quasi-spherical.
\end{abstract}

\section{Introduction}

The best known model of an accretion flow is the standard thin accretion disk developed by Shakura \& Sunyaev (1973), Novikov \& Thorne (1973), and Lynden-Bell \& Pringle (1974) (see Frank, King \& Raine (1992) for a detailed discussion). The basic assumption of the model is that the accreting gas is cool, compared to the local virial temperature, so that the flow acquires a thin disk configuration. The disk parameters are calculated assuming an equilibrium between the viscous energy generation inside the disk and radiative cooling from the surface; the latter is computed under the assumption that the disk is optically thick in the vertical direction.

The thin disk model has been widely and successfully used for modeling various low energy systems such as accreting white dwarfs and pre-main-sequence stars (Frank et al. 1992). However, the model has had less success explaining the characteristics of relativistic objects such as accreting black holes. There are essentially two problems: 1) In a system containing an accreting black hole, the accretion disk must extend inwards to the Schwarzschild radius, $R_{S c h w}$. However, for reasonable mass accretion rates and the standard $\alpha$ viscosity prescription, the Lightman-Eardley instability (Lightman \& Eardley 1974) causes a breakdown of the thin disk configuration at radii $R \sim R_{S c h w}$. Therefore, there is some doubt that a thin accretion disk can exist at all close to a black hole. 2) Even if a thin disk is viable, there is a problem with the spectrum. Since the gas in a thin disk model is optically thick, we would expect a roughly blackbody-like spectrum at the high frequency end, with a cutoff at several keV. However, extensive observations of black 
hole systems by GINGA, GRANAT, and GRO (Tanaka 1989, Grebenev et al. 1993, Maisack et al. 1993, Harmon et al. 1994, Johnson et al. 1994, Kinzer et al. 1994, Gilfanov et al. 1995) have made it clear that the spectra of most black hole accretors have a hard power-law components extending to few $\times 100 \mathrm{keV}$. This component has to be radiated by an optically thin plasma with temperature $T \gtrsim 10^{9} \mathrm{~K}$. The required temperature is at least an order of magnitude higher than that predicted by the standard thin disk model.

Shapiro, Lightman, \& Eardley (1976, hereafter SLE) discovered a new class of solutions at subEddington accretion rates, where the accretion flow is optically thin and quite hot $\left(T_{e} \sim 10^{8}-10^{9}\right.$ $\mathrm{K})$. These authors also introduced the important idea of a two-temperature plasma, in which ions are much hotter than electrons. Since the SLE solution has exactly the necessary characteristics (optically thin hot gas) to explain the spectra of accreting black holes, it has been widely studied and applied in models of X-ray binaries and active galactic nuclei (e.g. Kusunose \& Takahara 1985, 1989; White \& Lightman 1989; Wandel \& Liang 1991; Melia \& Misra 1993; Luo \& Liang 1994). Unfortunately, the SLE solution is thermally unstable (Pringle, Rees, \& Pacholczyk 1973, Piran 1978). The efficiency of bremsstrahlung cooling in the optically thin gas is proportional to the particle density which decreases with increasing temperature. Therefore, as the gas is perturbed to a higher temperature, the rate of cooling per unit mass decreases and the gas heats up even further in a runaway process. The thermal instability of the SLE solution makes it unlikely that real flows can take up this configuration.

Recently, a new class of two-temperature advection-dominated solutions has been discovered (Narayan \& Yi 1994, 1995a, 1995b; Abramowicz et al. 1995; Chen 1995; Chen et al. 1995). These advection-dominated solutions are optically thin, are hotter even than the SLE solution, and are viscously and thermally stable to large-wavelength perturbations (Abramowicz et al. 1995, Narayan \& Yi 1995b). Although Kato, Abramowicz \& Chen (1995) have discovered an instability in these flows at short wavelengths, they show that the mode amplitude does not grow significantly and so the viability of the solutions is not in doubt. The advection-dominated model has been fairly successful in explaining a number of low-luminosity systems (e.g. a model for Sagittarius A* by Narayan, Yi, \& Mahadevan [1995], a model for the soft X-ray transient A062000 by Narayan, McClintock \& Yi [1996], and a model for the central source in NGC 4258 by Lasota et al. [1996]) and is perhaps also a reasonable model for more luminous systems (Narayan 1996; Narayan, Yi, \& Mahadevan 1996).

The most detailed advection-dominated models considered so far are based on a two-temperature plasma which cools via bremsstrahlung, synchrotron and Comptonization processes. However, there have been discussions in the literature questioning whether a two temperature plasma can occur at all in astrophysical accretion flows (Phinney 1981, Rees et al. 1982). The discussion has been further fueled by the work of Begelman \& Chiueh (1988), who identified a particular mechanism involving collective plasma waves, which may under certain conditions pump energy directly into the electrons and bring the ions and electrons into thermal equilibrium. An important question therefore is the following: are there stable hot solutions, analogous to the two-temperature advection-dominated solution, if the plasma is well-coupled and has a single temperature for the ions and electrons? Abramowicz et al. (1995) and Chen (1995) did consider single-temperature plasmas in their studies of advection-dominated flows, but they included only bremsstrahlung cooling. As yet, one-temperature models with a more detailed cooling, including synchrotron radiation and Comptonization of synchrotron and bremsstrahlung photons, has not been considered, even though at $T \sim 10^{10} \mathrm{~K}$ with an equipartition magnetic field these processes completely dominate over pure bremsstrahlung cooling. This is the study we present here.

In $\S 2$ we derive the equations that describe an accreting one-temperature plasma. We then present the main results of the paper in $\S 3$. We find that, as in the two-temperature case (Narayan 
\& Yi 1995b, hereafter NY), the equations allow three branches of solutions: the standard thin Shakura \& Sunyaev disk, a hot optically-thin, thermally unstable solution, which is equivalent to the SLE branch for a two-temperature plasma, and finally a new hotter and thermally stable disk solution. We describe the properties of the new solution and compare it to the corresponding twotemperature solution branch. Among other things, we find that the one-temperature solution is hotter than the equivalent two-temperature one and is not advection-dominated for a wide range of accretion rates. This means that for a given $\dot{M}$ the one-temperature disk is considerably more luminous. We conclude in $\S 4$ with a summary and discussion.

\section{Basic Equations}

\subsection{One-Temperature Accretion Flow}

Narayan \& Yi (1994, 1995b) derived a set of local equations describing a vertically averaged, axisymmetric, two-temperature advection-dominated flow. Here we slightly modify these equations by setting the electron and ion temperatures equal to each other. We also include radiation pressure, which NY neglected.

The vertically averaged pressure $p$ and density $\rho$ of the accreting gas are given by:

$$
p=\rho c_{s}^{2}, \quad \rho=\frac{\dot{M}}{4 \pi R H|v|},
$$

where $c_{s}$ is the local isothermal sound speed, $\dot{M}$ is the mass accretion rate, $R$ is the radius, $H$ is the vertical scale height of the disk, and $v$ is the radial velocity. We write the total pressure as the sum of the gas, radiation and magnetic pressures, $p=p_{g}+p_{r}+p_{m}$, and we define three quantities that describe their relative magnitudes: $\beta=p_{g} / p$ determines the fraction of the total pressure due to gas pressure; $\beta_{m}=\left(p_{r}+p_{g}\right) / p=1-p_{m} / p$ determines the importance of magnetic field pressure; finally $\chi$ is defined such that $\beta=\chi \beta_{m}$ so that $\chi$ represents the ratio of gas pressure to the sum of gas and radiation pressure, $\chi=p_{g} /\left(p_{g}+p_{r}\right)$. For $\chi \sim 1$ radiation pressure is unimportant, and for $\chi \ll 1$ radiation pressure dominates over gas pressure. In our calculations we fix the value of $\beta_{m}$, typically at 0.5 corresponding to magnetic pressure equal to $50 \%$ of the total pressure, and then solve for $\chi$ and $\beta$.

With the above definitions, the scaled relations derived by NY take the form:

$$
\begin{aligned}
v & =-2.12 \times 10^{10} \frac{\alpha c_{1}}{\sqrt{r}} \mathrm{~cm} \mathrm{~s}^{-1}, \\
c_{s}^{2} & =4.5 \times 10^{20} \frac{c_{3}}{r} \mathrm{~cm}^{2} \mathrm{~s}^{-2}, \\
\rho & =3.79 \times 10^{-5} \frac{\dot{m}}{\alpha c_{1} m \sqrt{c_{3} r^{3}}} \mathrm{~g} \mathrm{~cm}^{-3}, \\
B & =6.55 \times 10^{8} \sqrt{\frac{\left(1-\beta_{m}\right) \dot{m}}{\alpha c_{1} m} \sqrt{\frac{c_{3}}{r^{5}}}} \mathrm{G}, \\
f & =\frac{1}{\epsilon^{\prime}}\left(\frac{5 / 3-\gamma}{\gamma-1}\right), \quad \gamma=\frac{32-24 \beta-3 \beta^{2}}{24-21 \beta}, \\
q^{+} & =1.84 \times 10^{21} \frac{\epsilon^{\prime} \dot{m} \sqrt{c_{3}}}{m^{2} r^{4}} \mathrm{erg} \mathrm{cm}^{-3} \mathrm{~s}^{-1} .
\end{aligned}
$$

Here $B$ is the magnetic field strength and the quantity $f$ determines the degree to which the flow is advection-dominated. $f$ ranges from 0 to 1 ; the limit of $f=1$ corresponds to the extreme case 
when all the energy is stored in the gas and advected, while $f \rightarrow 0$ corresponds to a standard cooling-dominated disk where most of the generated energy is radiated locally. The quantity $q^{+}$ is the viscous dissipation of energy per unit volume, and the factors $\epsilon^{\prime}, c_{1}$, and $c_{3}$ are given by

$$
\begin{aligned}
\epsilon^{\prime} & =\frac{1}{2}\left(\frac{18 \alpha^{2}-x^{2}}{2 x}-5\right), \quad x=\frac{9 c_{3} \alpha^{2}}{2}, \\
c_{1} & =\frac{3}{2} c_{3}, \\
c_{3} & =3.12 \times 10^{-13} \frac{T r}{\beta} .
\end{aligned}
$$

In the above equations masses are scaled in units of the solar mass

$$
M=m M_{\odot}
$$

accretion rates in Eddington units

$$
\dot{M}=\dot{m} \dot{M}_{E d d}, \quad \dot{M}_{E d d}=\frac{L_{E d d}}{\eta_{e f f} c^{2}}=\frac{4 \pi G M}{\eta_{e f f} \kappa_{e s} c}=1.39 \times 10^{18} \mathrm{mg} \mathrm{s}^{-1}
$$

where $\kappa_{e s}=0.4 \mathrm{~cm}^{2} \mathrm{~g}^{-1}$ and we assume the standard efficiency factor $\eta_{\text {eff }}=0.1$ (e.g. Frank et al. 1992); and radii in Schwarzschild units

$$
R=r R_{S c h w}, \quad R_{S c h w}=\frac{2 G M}{c^{2}}=2.95 \times 10^{5} \mathrm{mcm} .
$$

If we choose the values of $r, m, \dot{m}, \alpha$ and $\beta_{m}$, and assume specific values for $\beta$ and $T$, equations (11), (2) and (3) yield all the other disk parameters.

\subsection{Electron-Positron Pair Equilibrium}

Some of the hot solutions we calculate in this paper have temperatures of up to $10^{10}-10^{11} \mathrm{~K}$ (see Figure 6[d]), and it therefore becomes necessary to include in our model the effects of relativistic pair production and annihilation. Since a complete solution of the pair balance problem is beyond the scope of our calculation, we make some simplifying assumptions.

Svensson $(1982,1984)$ has explored the properties of pair equilibria in a uniform relativistic plasma where photons are generated via pair annihilation and bremsstrahlung. He finds that for temperatures below a certain critical value $T_{c}$ there are two equilibrium branches available that are characterized by the ratio $z$ of the pair number density, $n_{+}$, to the number density of protons, $n_{p}=n_{e}-n_{+}:(1)$ an optically thin low- $z$ branch $(z \ll 1)$ where pair production is dominated by particle-particle processes, and (2) a high- $z$ branch $(z \geq 1)$ where the Thomson scattering optical depth is of order unity and photon-photon pair production dominates. We confine our calculations to the low- $z$ branch where only particle-particle processes are important. Svensson has shown that the electron-electron pair production rate is always larger than the electronproton rate by a factor of $\sim 10$. This allows us to ignore the contribution from electron-proton collisions.

With the above simplifications, we can solve for the equilibrium pair density, $z$, analytically at any given temperature. For a thermal distribution of electrons and positrons, the pair annihilation rate per unit volume is (Svensson 1982):

$$
\left(\dot{n}_{+}\right)_{a n n}=\pi c r_{e}^{2} n_{e} n_{+} g(\theta)
$$


where $\theta=k_{B} T / m_{e} c^{2}$ is the dimensionless electron temperature, $r_{e}=e^{2} / m c^{2}$, and $g(\theta)$ is given by:

$$
g(\theta)=\left[1+\frac{2 \theta^{2}}{\ln (1.12 \theta+1.3)}\right]^{-1}
$$

For the electron-electron pair production rate we adopt the expression used by White \& Lightman (1989):

$$
\left(\dot{n}_{+}\right)_{e e}=\operatorname{cr}_{e}^{2} n_{e}^{2} \begin{cases}2 \times 10^{-4} \theta^{3 / 2} \exp (-2 / \theta)(1+0.015 \theta), & \text { if } \theta \ll 1, \\ (112 / 27 \pi) \alpha_{f}^{2}(\ln \theta)^{3}(1+0.058 / \theta)^{-1}, & \text { if } \theta \gg 1,\end{cases}
$$

where $\alpha_{f}$ is the fine structure constant. Local equilibrium requires that $\left(\dot{n}_{+}\right)_{e e}=\left(\dot{n}_{+}\right)_{\text {ann }}$, which allows us to solve for $z$ as a function of the plasma temperature. We find that for the highest temperature of interest, $T=10^{11} \mathrm{~K}, z$ has a maximum value $\sim 0.1$, and that it decreases monotonically in cooler plasmas. This implies that our low- $z$ assumption is consistent.

Given $z$ we can compute the total number density of electrons in the disk, $n_{e}=n_{p}(1+z)$, and number density of electrons plus positrons, $n_{ \pm}=n_{p}(1+2 z)$.

\subsection{Cooling Processes}

Since ions are considerably more massive than electrons and positrons, viscous heating will affect primarily the ions (SLE, Phinney 1981, Rees et al. 1982), which will then transfer this energy to the electrons. In their paper NY assume that the energy transfer occurs only via Coulomb interactions, ignoring possible non-thermal coupling mechanisms (e.g. Begelman \& Chiueh 1988). Since Coulomb interactions are not efficient at low densities, the ions are always hotter than the electrons. In this paper we assume that the ions and electrons have been brought into thermal equilibrium by some process, without explicitly specifying the actual coupling mechanism.

The cooling of the hot plasma occurs mainly through the electrons and positrons. In our model we consider three cooling processes: bremsstrahlung, synchrotron, and Compton cooling by both bremsstrahlung and synchrotron photons.

Bremsstrahlung Cooling The free-free radiation in a plasma is produced through five different types of interactions: electron-electron $\left(e^{-} e^{-}\right)$, electron-ion $\left(e^{-} i\right)$, positron-electron $\left(e^{-} e^{+}\right)$, positron-positron $\left(e^{+} e^{+}\right)$, and positron-ion $\left(e^{+} i\right)$. However, $e^{-} i$ and $e^{+} i$ processes can be combined together since their corresponding cooling rates are identical, and the same is true for $e^{-} e^{-}$ and $e^{+} e^{+}$processes. Thus the total bremsstrahlung cooling rate per unit volume is just the sum of the rates for $e^{ \pm} i, e^{ \pm} e^{ \pm}$, and $e^{-} e^{+}$processes; and we can write $q_{b r}^{-}=q_{e i}^{-}+q_{e e}^{-}+q_{+-}^{-}$, provided we replace $n_{p} n_{e}$ by $n_{p} n_{ \pm}$and $n_{e}^{2}$ by $\left(n_{e}^{2}+n_{+}^{2}\right)$ in our calculations of $q_{e i}^{-}$and $q_{e e}^{-}$respectively.

Following NY, we adopt the expressions from Stepney \& Guilbert (1983) and Svensson (1982) for the $e^{ \pm} i, e^{ \pm} e^{ \pm}$, and $e^{-} e^{+}$bremsstrahlung cooling rates:

$$
\begin{aligned}
q_{e i}^{-} & =1.48 \times 10^{-22} n_{p} n_{ \pm} F_{e i} \mathrm{erg} \mathrm{cm}^{-3} \mathrm{~s}^{-1} ; \\
q_{e e}^{-} & = \begin{cases}2.56 \times 10^{-22}\left(n_{e}^{2}+n_{+}^{2}\right) \theta^{1.5}\left(1+1.1 \theta+\theta^{2}-1.25 \theta^{2.5}\right) \mathrm{erg} \mathrm{cm}^{-3} \mathrm{~s}^{-1}, & \text { if } \theta<1, \\
3.42 \times 10^{-22}\left(n_{e}^{2}+n_{+}^{2}\right) \theta(\ln (1.123 \theta)+1.28) \mathrm{erg} \mathrm{cm}^{-3} \mathrm{~s}^{-1}, & \text { if } \theta \geq 1 ;\end{cases} \\
q_{+-}^{-} & = \begin{cases}3.43 \times 10^{-22} n_{e} n_{+}\left(\theta^{0.5}+1.7 \theta^{2}\right) \mathrm{erg} \mathrm{cm}^{-3} \mathrm{~s}^{-1}, & \text { if } \theta<1, \\
6.84 \times 10^{-22} n_{e} n_{+} \theta(\ln (1.123 \theta)+1.24) \mathrm{erg} \mathrm{cm}^{-3} \mathrm{~s}^{-1}, & \text { if } \theta \geq 1 ;\end{cases}
\end{aligned}
$$

where

$$
F_{e i}= \begin{cases}4 \sqrt{\frac{2 \theta}{\pi^{3}}}\left(1+1.781 \theta^{1.34}\right), & \text { if } \theta<1 \\ \frac{9 \theta}{2 \pi}(\ln (1.123 \theta+0.48)+1.5), & \text { if } \theta \geq 1\end{cases}
$$


Synchrotron Cooling Synchrotron cooling is very important in our model, both because we assume an equipartition magnetic field in the disk and because we consider relativistic temperatures for the electrons and positrons.

Pacholczyk (1970) has calculated the spectrum of synchrotron emission by a relativistic Maxwellian distribution to be

$$
\epsilon_{s} d \nu=4.43 \times 10^{-30} \frac{4 \pi \nu n_{ \pm}}{K_{2}(1 / \theta)} I\left(\frac{x_{M}}{\sin \phi}\right) d \nu \operatorname{erg~cm}^{-3} \mathrm{~s}^{-1},
$$

where

$$
x_{M}=\frac{2 \nu}{3 \nu_{0} \theta^{2}}, \quad \nu_{0}=\frac{e B}{2 \pi m_{e} c}
$$

$\phi$ is the angle between the velocity vector of the electrons and the direction of the local magnetic field, and $I(x)$ is a tabulated function. Positrons produce synchrotron radiation at the same rate as electrons do and therefore in equation (11) we have used the total number density, $n_{ \pm}$, instead of $n_{e}$.

For an isotropic velocity distribution we can average $I\left(x_{M} / \sin \phi\right)$ over $\phi$ to get a new function $I^{\prime}\left(x_{M}\right)$ for which a fitting function was found by Mahadevan, Narayan \& Yi (1996):

$$
I^{\prime}\left(x_{M}\right)=\frac{4.0505}{x_{M}^{1 / 6}}\left(1+\frac{0.40}{x_{M}^{1 / 4}}+\frac{0.5316}{x_{M}^{1 / 2}}\right) \exp \left(-1.8899 x_{M}^{1 / 3}\right) .
$$

We substitute this function for $I\left(x_{M} / \sin \phi\right)$ in equation (11).

Equations (11) and (12) are valid only for optically thin emission. However, below some critical frequency $\nu_{c}$ the emission becomes self-absorbed and that has to be taken into account in computing the total cooling rate. We estimate $\nu_{c}$ as the frequency at which the synchrotron emission from a thin annulus of height $2 H$, radius $R$, and thickness $\Delta R$ is equal to the blackbody emission (in the Rayleigh-Jeans limit) from the upper and lower surfaces of the annulus (NY presented a similar argument but they used a spherical rather than cylindrical geometry). This condition gives us the equation:

$$
2 H \cdot 2 \pi R \Delta R \cdot \epsilon_{s} d \nu=2 \cdot 2 \pi R \Delta R \cdot 2 \pi \frac{\nu_{c}^{2}}{c^{2}} k T d \nu
$$

which we can solve numerically for given values of $R, H$, and $T$ to obtain $\nu_{c}$.

Given the value of the critical frequency, we can estimate the total synchrotron emission per unit volume as follows. We assume that for frequencies below $\nu_{c}$ the emission is completely selfabsorbed so that the volume emissivity can be approximated by the blackbody emission from the surface of the disk divided by the disk volume. Above $\nu_{c}$ the emission is optically thin and equation (11) can be used. To get the total cooling per unit volume by synchrotron emission, we then integrate over frequency:

$$
\begin{aligned}
& q_{s}^{-}=\frac{2 \pi R^{2}}{2 H \pi R^{2}} \int_{0}^{\nu_{c}} 2 \pi \frac{\nu^{2}}{c^{2}} k T d \nu+\int_{\nu_{c}}^{\infty} \epsilon_{\nu} d \nu \\
& =\frac{2 \pi k T \nu_{c}^{3}}{3 H c^{2}}+6.76 \times 10^{-28} \frac{n_{ \pm}}{K_{2}(1 / \theta) a^{1 / 6}}\left[\frac{1}{a_{4}^{11 / 2}} \Gamma\left(\frac{11}{2}, a_{4} \nu_{c}^{1 / 3}\right)+\right. \\
& \left.+\frac{a_{2}}{a_{4}^{19 / 4}} \Gamma\left(\frac{19}{4}, a_{4} \nu_{c}^{1 / 3}\right)+\frac{a_{3}}{a_{4}^{4}}\left(a_{4}^{3} \nu_{c}+3 a_{4}^{2} \nu_{c}^{2 / 3}+6 a_{4} \nu_{c}^{1 / 3}+6\right) e^{-a_{4} \nu_{c}^{1 / 3}}\right] \text {, }
\end{aligned}
$$


where the parameters $a_{1}, a_{2}, a_{3}, a_{4}$ are defined as

$$
a_{1}=\frac{2}{3 \nu_{0} \theta^{2}}, \quad a_{2}=\frac{0.4}{a_{1}^{1 / 4}}, \quad a_{3}=\frac{0.5316}{a_{1}^{1 / 2}}, \quad a_{4}=1.8899 a_{1}^{1 / 3} .
$$

Compton Cooling Since our solutions are hot, Comptonization of soft photons by hot electrons becomes an important cooling mechanism. It is an especially important process for higher values of the accretion rate, when the scattering depth is of order unity and synchrotron emission is the dominant photon production mechanism, i.e. most photons are initially very soft.

We compute the Compton cooling rate using the Comptonized energy enhancement factor $\eta$, which is defined as the average ratio of the energy of a photon at escape to its initial energy. We adopt the following prescription for $\eta$, which is derived in Appendix A:

$$
\eta=e^{s(A-1)}\left[1-P\left(j_{m}+1, A s\right)\right]+\eta_{\max } P\left(j_{m}+1, s\right),
$$

where $P(a, x)=[1 / \Gamma(a)] \int_{0}^{x} t^{a-1} e^{-t} d t$ is the incomplete gamma function and

$$
\begin{aligned}
A & =1+4 \theta+16 \theta^{2}, \\
s & =\tau_{e s}+\tau_{e s}^{2}, \\
j_{m} & =\ln \left(\eta_{\max }\right) / \ln (A), \\
\eta_{\max } & =\frac{3 k T}{h \nu} .
\end{aligned}
$$

The parameter $A$ is the average increase in energy of a soft photon per scattering for a Maxwellian distribution of electrons (and positrons) at temperature $\theta, \tau_{e s}=2 n_{ \pm} \sigma_{T} H$ is the Thomson optical depth (counting both electrons and positrons), $j_{m}$ is the number of scatterings required for the maximum possible energy enhancement $\eta_{\max }$, and $\nu$ is the initial photon frequency. By definition, the Comptonized flux is the original flux multiplied by $\eta$.

Since the synchrotron spectrum is strongly peaked at the critical frequency $\nu_{c}$, we estimate the cooling rate due to the Comptonization of the synchrotron radiation simply by computing $\eta$ for $\nu=\nu_{c}$ :

$$
q_{C, s}^{-}=\eta\left(\nu_{c}\right) q_{s}^{-} .
$$

The spectrum of bremsstrahlung radiation is practically flat for frequencies between the bremsstrahlung self-absorption frequency, $\nu_{b r}$, and the exponential cutoff at $\nu=k T / h$. The emission at frequencies $\nu<\nu_{b r}$ is negligible compared with the integrated bremsstrahlung cooling rate. Therefore, we can approximate free-free emission per unit frequency as the ratio $q_{b r}^{-} /\left(\frac{k T}{h}-\nu_{b r}\right)$. We estimate $\nu_{b r}$ as the frequency at which the free-free absorption opacity, $\kappa_{\nu}^{f f}$ (which is frequency dependent) is equal to the electron scattering opacity, $\kappa_{e s}$. Below $\nu_{b r}$ Comptonization is not important, since absorption dominates over scattering. Therefore to calculate the Comptonization enhancement we perform the following numerical integration:

$$
q_{C, b r}^{-}=\int_{\nu_{b r}}^{\frac{k T}{h}} \eta(\nu) \frac{d q_{b r}^{-}}{d \nu} d \nu=\int_{\nu_{b r}}^{\frac{k T}{h}} \eta(\nu) \frac{q_{b r}^{-}}{\frac{k T}{h}-\nu_{b r}} d \nu .
$$

Cooling of the Optically Thick Gas The total cooling rate for the optically thin gas is simply the sum of the two components calculated above:

$$
q^{-}=q_{C, b r}^{-}+q_{C, s}^{-}
$$


This rate is appropriate for the hot solutions, which are generally optically thin. However, to reproduce the equilibrium solution corresponding to the cool optically thick Shakura \& Sunyaev disk, we use the generalized cooling formula given by NY:

$$
q^{-}=\frac{4 \sigma T^{4} / H}{1.5 \tau+\sqrt{3}+\frac{4 \sigma T^{4}}{H}\left(q_{C, b r}^{-}+q_{C, s}^{-}\right)^{-1}},
$$

where $\tau=\tau_{e s}+\tau_{a b s}$ is the total optical depth of the disk in the vertical direction and $\tau_{a b s}=$ $\left(H / 4 \sigma_{B} T^{4}\right)\left(q_{C, b r}^{-}+q_{C, s}^{-}\right)$is the optical depth for absorption. For a small optical depth, equation (21) reduces to equation (20), and therefore this formula can be used in the limit of both very high and very low optical depth.

Radiation Pressure The total cooling rate allows us to compute the radiation pressure in the disk,

$$
p_{r}=\frac{q^{-} H}{2 c}\left(\tau+\frac{2}{\sqrt{3}}\right) .
$$

The gas pressure in the accreting flow is simply:

$$
p_{g}=\rho k T\left(\frac{1}{\mu_{i} m_{u}}+\frac{1}{\mu_{e} m_{u}}\right)
$$

where $\mu_{i}$ and $\mu_{e}$ are the effective molecular weights of the ions and electrons, given by

$$
\mu_{i}=\frac{4}{1+3 X}=1.23, \quad \mu_{e}=\frac{2}{1+X}=1.14,
$$

with numerical values computed for a hydrogen mass fraction $X=0.75(\mathrm{NY})$. Given $p_{r}$ and $p_{g}$, the parameter $\chi$, defined in $\S 2.1$, is readily computed:

$$
\chi=\beta / \beta_{m}=p_{g} /\left(p_{r}+p_{g}\right) .
$$

\section{Properties of the Solutions}

An equilibrium thermal state for the accretion flow requires that viscous heating minus the advected energy is exactly balanced by the total radiative cooling at each radius:

$$
q^{+}(1-f)=q^{-} .
$$

For fixed values of $m, r, \dot{m}$, and $\beta_{m}$, the equations (1)-(26) comprise a closed set which can be solved to obtain all the relevant parameters of the accretion flow. We solve the equations numerically using $T$ and $\chi$ as free variables. We begin by assuming arbitrary values for $T$ and $\chi$ and use equations (2)-(6) to calculate all the parameters of the plasma, including the pair fraction. Then we use equations (7)-(24) to calculate the cooling rate $q^{-}$and the gas and radiation pressures, $p_{g}$ and $p_{r}$. Now we compute $\chi=p_{g} /\left(p_{r}+p_{g}\right)$ (see equation (25)) and compare the result with the initially assumed value. If the two values are not equal, we vary $\chi$ keeping $T$ fixed until there is agreement between the initial and final $\chi$. We then vary $T$, optimizing $\chi$ at each step, until the energy equation (26) is satisfied. At this point we have a self-consistent equilibrium solution. 
All calculations presented below were done for an equipartition magnetic field strength in the disk, $\beta_{m}=0.5$. However, we have found that models with $\beta_{m}=0.95$ (where magnetic pressure constitutes only $5 \%$ of the total pressure) produce practically the same results.

Figure 11 illustrates the variations with $T$ of the two sides of equation (26); the solid line shows $q^{+}(1-f)$ and the dashed line shows the total radiative cooling $q^{-}$. These results are for a single-temperature accretion disk of mass accretion rate $\dot{M}=10^{-5} \dot{M}_{E d d}$ around a black hole of mass $M=10 M_{\odot}$ at radial distance $R=10 R_{S c h w} ; \chi$ has been optimized at each $T$. The three points where the two curves intersect, labeled as 1, 2, and 3 on the figure, correspond to three equilibrium states of the accretion disk where the energy balance equation (26) is satisfied. The equilibrium point 1 corresponds to the standard Shakura \& Sunyaev (1973) thin disk; the middle point is the one-temperature equivalent of the unstable Shapiro, Lightman, \& Eardley (1976) solution; the equilibrium point labeled 3 is a new hot solution which which does not seem to have been discussed before in the literature. It is related to the advection-dominated one-temperature solution of Abramowicz et al. (1995), but with some important differences as we argue later.

Note that the slope of the cooling curve in Figure 1 changes twice. At $T \approx 10^{6.2} \mathrm{~K}$ the change of slope indicates a transition from an optically thick to an optically thin flow. There is a corresponding kink in the heating curve, since at this point the cooling rate is at its maximum and radiation pressure briefly dominates over the gas pressure, causing a sharp decrease in gas density. For $T<10^{6.2} \mathrm{~K}$, the gas cools as a blackbody and the cooling rate increases with increasing temperature. For $T>10^{6.2} \mathrm{~K}$, the dominant cooling process is optically thin bremsstrahlung, which is less efficient at higher temperatures. At $T \approx 10^{9.5} \mathrm{~K}$ the slope of $\log \left(q^{-}\right)$changes again, indicating a point where synchrotron radiation becomes the dominant cooling process. In contrast to bremsstrahlung, the synchrotron emission increases with increasing temperature, hence the positive slope of the cooling curve at these high temperatures.

By inspection it is obvious that equilibria 1 and 3 are thermally stable. In both of these equilibria, if the gas is perturbed to a higher temperature, the rate of cooling becomes greater than the rate of heating, allowing the medium to cool back to the equilibrium value of $T$. Similarly, if $T$ becomes slightly smaller, the heating rate exceeds the cooling rate and the gas heats up back to the equilibrium temperature. The equilibrium 2 on the other hand is unstable. Here a small increase or decrease in $T$ leads to a runaway situation where $T$ deviates progressively more rapidly from the equilibrium value.

We have computed the properties of the accreting gas corresponding to the three equilibria for different values of $\dot{M}$. The resulting curves for a central black hole of mass $M=10 M_{\odot}$ at fixed radial distance $R=10 R_{S c h w}$ are plotted in Figure 2 for $\alpha=0.1$ (heavy line) and $\alpha=1.0$ (thin line). The curves are labeled 1,2, and 3 to indicate correspondence with the equilibria labeled 1, 2, 3 in Figure 1. The six panels in Figure 2 show how various physical properties of the solutions depend on the accretion rate. The unstable SLE solution (branch 2) is indicated by a dashed line.

For $\alpha=0.1$ the topology of our results is qualitatively similar to that of the corresponding solutions for two-temperature disks described by Chen et al. (1995). We see that the two hotter branches merge together at some critical accretion rate $\left(\dot{M}_{\text {crit }}\right)$, above which the cooling in the disk is so efficient that the only equilibrium allowed is the standard thin disk solution. For $\alpha=1$ the hot branch extends to much higher values of the accretion rate; in fact, the value of $\dot{M}_{\text {crit }}$ goes up by $\sim 2$ orders of magnitude, in agreement with the results of Abramowicz et al. (1995) and NY. Somewhat surprisingly, the topology of the solution curves for $\alpha=1$ continues to be similar to that for $\alpha=0.1$. This is different from the result reported by Chen et al. (1995), who found that for $\alpha=1$ the middle branch merged with the cold disk solution, whereas the hottest branch extended independently upward to become the optically-thick advection- 
dominated solution discovered by Abramowicz et al. (1988). The value of the critical $\alpha$ at which the topology changes is, however, sensitive to the details of the radiation processes included in the model (M. Abramowicz, private communication). In fact, we do find the same topology change in our results when we push $\alpha$ above $\sim 1.7$.

For both $\alpha=0.1$ and $\alpha=1$, the curves corresponding to the three equilibria in Figure 2(a) have positive slopes, i.e. $\partial \dot{M} / \partial \Sigma>0$. This implies that all three solutions are viscously stable (cf. Frank, King, \& Raine 1992, p.103). Note also that our new hot solution is optically thin for all values of $\dot{M}$ where the solution exists (Figure 2[b]) and cools mainly through synchrotron and Comptonized synchrotron processes (Figure 2[ $[\mathrm{c}]$ ).

Figure 3 directly compares the two-temperature (thin line) and one-temperature (heavy line) equilibrium solutions for a disk with $\alpha=0.1$. For temperatures below $T \approx 10^{9} \mathrm{~K}$ the two curves merge, since the electron and ion temperatures in the two-temperature solution are nearly equal (Figure $3[\mathrm{c}]$ ) and therefore, two-temperature and one-temperature solutions are effectively the same. However, for $T \gtrsim 10^{9} \mathrm{~K}$ the two solutions differ significantly. The most important distinction is in the value of the advected energy fraction $f$ (Figure 3[b]). For the hot twotemperature solution, we have $f \rightarrow 1$ at all $\dot{M}$, showing that this branch is always advectiondominated. In contrast, we see that our one-temperature hot solution is cooling-dominated $(f<0.1)$ for a wide range of accretion rates. This is a completely new result, as prior to this the only hot stable solutions known were all advection-dominated (NY, Abramowicz et al. 1995, Chen 1995, Chen et al. 1995). Since the cooling rate is given by $q^{-}=q^{+}(1-f)$, we see that, at a given accretion rate, our single-temperature disk with its low value of $f$ is considerably more luminous than the equivalent two-temperature flow.

In the case of the two-temperature advection-dominated hot solutions discussed by NY and Abramowicz et al. (1995), the thermal stability of the solution to long-wavelength perturbations has been shown to be the direct result of advection. However, our hot one-temperature solution branch 3 is cooling-dominated and has very little advection. Why is this solution stable? The answer is obvious from Figure 1, where we see that the introduction of synchrotron cooling causes the cooling curve $q^{-}(T)$ to be steeply positive for $T>10^{9.5} \mathrm{~K}$. Therefore, thermal stability is achieved in this solution because of the usual reason, namely that the cooling increases more rapidly than the heating. Note that we would not have obtained this result if we had included only bremsstrahlung cooling. In fact, Abramowicz et al. (1995) considered the pure bremsstrahlung one-temperature case and they obtained a hot advection-dominated solution. Thus, the inclusion of synchrotron cooling has an important effect on the basic physics of the accretion flow. Although a full linear stability analysis along the lines of Kato et al. (1995) has not been done on our new solutions, we suspect that these solutions are stable to all linear perturbations, regardless of wavelength.

Figure 1 illustrates the radial structure of a disk with $\alpha=0.1$ for a fixed value of the accretion rate, $\dot{M}=10^{-4} \dot{M}_{E d d}$. The inner edge was chosen to be at $R=3 R_{S c h w}$, corresponding to the last stable orbit. As expected, we see that the two-temperature (thin line) and one-temperature (heavy line) solutions merge at large radii where the temperature is low and even the twotemperature plasma has effectively a single temperature (see Figure $₫[\mathrm{c}]$ ). Thus, away from the center, the flow is advection-dominated (see Figure $\mathbb{Q}[\mathrm{b}]$ ) and almost spherical with $H / R \sim 1$ (Figure $\mathbb{A}[\mathrm{d}]$ ), regardless of whether we have a two-temperature or one-temperature solution. However, closer to the accreting object, the flow becomes increasingly cooling-dominated in the one-temperature case and settles into a disk with $H / R \sim 0.15$ at the inner edge. The resulting geometry - thinnish disk near the inner edge and quasi-spherical flow at larger radii - is unusual for accretion disk models. Another interesting feature of our one-temperature solution is that the temperature profile of the disk is not monotonic but has a well defined maximum at $R \sim 40 R_{S c h w}$. 
Therefore, the hottest part of the spectrum does not come from the inner edge of the disk.

NY showed that their advection-dominated hot two-temperature solution exists only for accretion rates $\dot{M}$ less than a critical rate $\dot{M}_{\text {crit }}$, which depends on $\alpha$ and $R$ (see also Abramowicz et al. 1995). We find a similar result also in the case of our cooling-dominated hot one-temperature solution. Figure 5 shows the critical accretion rate $\dot{M}_{\text {crit }}$ for our solution as a function of $R$ as well as the variation of the gas temperature. The two panels on the left (Figure $5[\mathrm{a}, \mathrm{b}]$ ) compare the results for the one-temperature and two-temperature solutions for $\alpha=0.1$. We see that the electron temperatures for the two solutions are nearly equal, but the behavior of $\dot{M}_{\text {crit }}$ is quite different in the two cases. For a two-temperature flow, $\dot{M}_{\text {crit }}$ is roughly independent of $R$ for $R \lesssim 10^{3} R_{S c h w}$ and is given approximately by $\dot{M} \sim 0.3 \alpha^{2} \dot{M}_{E d d}$ (see NY and Abramowicz et al. 1995 for a discussion of the $\alpha^{2}$ scaling). In contrast, $\dot{M}_{\text {crit }}$ decreases almost linearly with decreasing $R$ in the one-temperature case, so that $\dot{M} \sim 310^{-3} \alpha^{2}\left(R / R_{S c h w}\right) \dot{M}_{E d d}$ for $R \lesssim 10^{3} R_{S c h w}$ Thus, near the black hole, $\dot{M}_{\text {crit }}$ for the one-temperature solution is smaller than that of the two-temperature solution by as much as two orders of magnitude.

The steep decrease of $\dot{M}_{\text {crit }}$ with decreasing $R$ leads to an interesting effect which is already seen in Figure 1 . For a wide range of $\dot{M}$, it is possible to have $\dot{M}<\dot{M}_{\text {crit }}$ at large radii, but to have $\dot{M}>\dot{M}_{\text {crit }}$ at small radii, with a transition at some critical radius $R_{m i n}$. For the example shown in Figure 1, we have $R_{\text {min }}=5 R_{S c h w}$. In this case, we can have a hot one-temperature solution for $R>R_{\text {min }}$, but the solution disappears at $R=R_{\text {min }}$. Below $R_{\text {min }}$, the only equilibrium solution available is the cool thin accretion disk. (Note that, because of the very low $\dot{M} \sim 10^{-4} \dot{M}_{E d d}$, the Lightman-Eardley instability does not affect the thin disk solution in this case.) Thus, the example shown in Figure 1 has an extremely unusual structure. For $3 R_{S c h w}<R<5 R_{S c h w}$, there is a standard thin disk. Then, for $5 R_{S c h w}<R<40 R_{S c h w}$, we have a hot, but cooling-dominated thickish disk. Finally, for $R>40 R_{S c h w}$, we have a regular advection-dominated quasi-spherical flow. There are several discussions in the literature of models where an outer thin disk makes a transition to a hot inner flow (e.g. SLE, Wandel \& Liang 1991, Narayan, McClintock, \& Yi 1996, Narayan 1996), but here we have an example of a model which does the opposite, namely transforms from an outer hot advection-dominated flow via a cooling-dominated hot flow to an inner thin disk. (See Melia 1994 for the closest example discussed previously in the literature.)

Figure 6 shows how the properties of the flow change as the accretion rate is reduced. There are some interesting effects. First, with decreasing $\dot{M}$, the critical radius $R_{\text {min }}$ disappears so that there is no longer any reason for the gas to make a transition to a cool disk on the inside. As $\dot{M}$ decreases still farther, even the cooling-dominated zone of the hot disk disappears, and advection becomes more important throughout the flow. Consequently the disk thickens considerably, and the maximum in the temperature profile at large radius becomes less apparent. For $\alpha=0.1$ and $\dot{M} / \dot{M}_{E d d}<10^{-8}$ the flow is advection-dominated and nearly virial at all radii.

Figure 6 $6[\mathrm{c}]$ shows the relative importance of Comptonized synchrotron cooling as a function of $R$ at various $\dot{M}$. We see that synchrotron and Comptonized synchrotron cooling is dominant in the inner regions, $R<100 R_{S c h w}$, regardless of the accretion rate. In the outer disk, on the other hand, where the flow is cooler and more advection-dominated, the cooling is mostly due to bremsstrahlung emission.

Though one-temperature solutions are restricted to smaller values of $\dot{M}$ than the corresponding two-temperature solutions, nevertheless we should note that for any given $\dot{M}$ they are significantly more luminous. This is because these flows are cooling-dominated at small radii where most of the radiation is emitted. Figure \&(a) illustrates this luminosity increase in the case of $\alpha=0.3$. Here we plot the total disk luminosity as a function of $\dot{M}$ for one-temperature (solid line) and two-temperature (dashed line) solutions. Note that at $\dot{M}=10^{-4} \dot{M}_{E d d}$ the one-temperature disk is $\sim 10^{4}$ times more luminous than its two-temperature counterpart. 
Figure $7(\mathrm{~b})$ shows how the total luminosity of our one-temperature solutions changes with $\alpha$. For a given value of $\dot{M}$, higher- $\alpha$ solutions are less luminous, since they are more advectiondominated (see Figure 2[e]). However, higher values of $\alpha$ allow the hot solution to survive up to higher accretion rates (see Figure 5[c]) with correspondingly higher luminosities. Thus, the most luminous system that we can model as a hot one-temperature disk extending down to $R=3 R_{S c h w}$ requires $\alpha=1.0$ and $\dot{M}=10^{-3} \dot{M}_{E d d}$, and has a total luminosity $L=10^{-2.8} L_{E d d}$. We could in principle use higher values of $\dot{M}$, but then as discussed above, we will have to include a cool thin disk at small radii $\left(R<R_{\min }\right)$.

\section{Discussion}

Most of the work done so far on modeling hot accretion disks has been based on two-temperature flows, where the electrons are much cooler than the ions (SLE; Rees et al. 1982; Melia \& Misra 1993; Narayan \& Yi 1995b; Narayan et al. 1995, 1996). However, if there exist mechanisms of energy transfer between ions and electrons that are more efficient than Coulomb scattering (Phinney 1981, Rees et al. 1982, Begelman \& Chiueh 1988), the plasma might be well-coupled and the ions and electrons may have the same temperature. In this paper we have investigated how the properties of the hot, stable two-temperature solution described by NY and Chen et al. (1995) are altered if ions and electrons in the accreting plasma are constrained to have the same temperature.

We start from the set of equations developed by NY, adding radiation pressure and particleparticle pair production processes, and improving some of the radiative cooling calculations. We solve these equations for various choices of the viscosity parameter, $\alpha$, and magnetic pressure parameter, $\beta_{m}$, explicitly setting the ion and electron temperatures equal to each other. The resulting three branches of solutions which we obtain (Figure 作) correspond to the standard thin disk (Shakura \& Sunyaev 1973, Novikov \& Thorne 1973, Lynden-Bell \& Pringle 1974), the one-temperature equivalent of the unstable hot solution discovered by SLE, and finally a new thermally stable hot solution whose properties we discuss in detail (§3). The new solution, which is identified by label 3 in Figures 1 and 2, is the one-temperature equivalent of the twotemperature advection-dominated solutions discussed by NY (see also Abramowicz et al. 1995, Chen et al. 1995).

For a viscosity parameter $\alpha=0.1$, we find that the topological relationships among the three solution branches are similar to those found by Chen et al. (1995) and NY for the twotemperature solutions. Specifically, we see that the two hot branches merge at some maximum value of the mass accretion rate, which we call $\dot{M}_{\text {crit }}$, such that for $\dot{M}>\dot{M}_{\text {crit }}$ the only solution available to the flow is the standard thin Shakura \& Sunyaev disk configuration. At higher $\alpha$, we confirm the result of Chen et al. (1995) that the topology changes. However, the critical $\alpha$ where the change occurs is 1.7 (at $R=10 R_{S c h w}$ ) for the one-temperature case we consider, rather then 0.3 in Chen et al. (1995).

We have computed the value of the critical mass accretion rate $\dot{M}_{\text {crit }}$ as a function of radius for different values of $\alpha$ (Figure 5[c]) and $\beta_{m}$. We find that $\dot{M}_{\text {crit }}$ varies roughly as $\alpha^{2}$ (Abramowicz et al. $1995, \mathrm{NY}$ ) and is relatively independent of $\beta_{m}$. A new result is that $\dot{M}_{\text {crit }}$ varies almost linearly with $R$ for $R \lesssim R_{S c h w}$, since in a two-temperature flow $\dot{M}_{\text {crit }}$ is roughly independent of $R$ in that region. The maximum value of the accretion rate at which the hot solution exists near the inner disk radius $\left(R=3 R_{S c h w}\right)$ is $\dot{M}_{c r i t} \sim 3 \times 10^{-3} \alpha^{2} \dot{M}_{E d d}$. In comparison, two-temperature hot solutions have $\dot{M}_{\text {crit }} \sim 0.3 \alpha^{2} \dot{M}_{E d d}$.

Though our single-temperature solutions are limited to lower mass accretion rates than those 
allowed by two-temperature models, this is compensated to some degree by strong cooling in the inner regions, where $f<0.1$ for $\dot{M}>10^{-3} \dot{M}_{\text {crit }}\left(3 R_{S c h w}\right.$ ) (Figure $5[\mathrm{f}]$ ). As a result, over the range of mass accretion rates, $10^{-3}<\dot{M} / \dot{M}_{\text {crit }}\left(3 R_{\text {Schw }}\right)<1$, our solutions are significantly more luminous than the corresponding two-temperature solutions (Figure $7[\mathrm{~b}]$ ).

This work represents the first example of a hot thermally stable accretion solution which is also cooling-dominated and therefore an efficient radiator. Because advection plays a very minor role in our new solutions, the thermal stability is not the result of advection as in the equivalent two-temperature solutions (NY, Abramowicz et al. 1995) or in the pure bremsstrahlung onetemperature solutions (Abramowicz et al. 1995). We show that the thermal stability of the hot one-temperature solution is primarily the result of including synchrotron emission, which leads to a rapid increase of the cooling rate with increasing temperature (see Figure 1).

An interesting feature of our models is the unusual geometry of the flow. Since $\dot{M}_{\text {crit }}$ decreases with decreasing $R$ in the inner parts of the disk (see Figure f), for a wide range of $\dot{M}$ the disk has a very unusual structure. In the inner regions, where $\dot{M}>\dot{M}_{\text {crit }}(R)$, the only possible solution for the accreting gas is the Shakura \& Sunyaev thin disk. Note that since we are restricted to relatively low mass accretion rates, the thin disk is viscously stable even at $R \sim 3 R_{S c h w}$. Further out, the hot solution exists, but it is cooling-dominated and therefore has a disk-like geometry. Finally, in the outer parts of the disk the flow is fully advection-dominated and becomes nearly quasi-spherical. This interesting result, however, presents a problem for our calculations. The inner radiative zone of the disk is cooler than the outer advection-dominated zone, so the relatively soft photons emitted on the inside will Compton cool the hot outer gas in a way that is incompatible with the local treatment of the radiative processes which we have used in this paper. The effects of the non-local radiation field need to be incorporated self-consistently into future calculations.

Since the gas temperatures in our solutions are on the order of $T \sim 10^{10} \mathrm{~K}$, pair production and annihilation processes must be taken into account. In our calculations we have included particle-particle pair production rates (White \& Lightman 1989) which give values for the pair fraction $z$ less than a few percent, but we neglected photon-photon and photon-particle processes. We believe that this treatment is justified for low values of $\dot{m}$ (low optical depth), since bremsstrahlung radiation and Comptonization become unimportant and the cooling is dominated by soft synchrotron photons which are incapable of producing pairs. However, for $\dot{M} \gtrsim 0.1 \dot{M}_{\text {crit }}$, Comptonization of synchrotron radiation begins to dominate and photon processes may become important. To place a limit on these processes we computed the equilibrium pair density taking into account photon-photon and photon-particle pair production in the Wien peak of the Comptonized spectrum, using the rates derived by Svensson (1984). The resulting values of $z$ are significantly higher than the values computed taking into account only particle-particle pair production, as $\dot{M}$ approaches $\dot{M}_{\text {crit }}$. However, even at $\dot{M}=\dot{M}_{\text {crit }}$, the maximum value of $z$ that we find with the inclusion of photon interactions is only $\sim$ few\%. Kusunose (1996) and Abramowicz (1996) confirm that pair processes are unimportant in the hot optically thin flows which they have studied. Thus, we believe that our treatment of pair processes is reliable in the entire parameter space we have considered.

This work was supported in part by NSF grant AST 9423209 to the Center for Astrophysics. RN thanks the Institute for Theoretical Physics (NSF grant PHY 9407194) for hospitality. IY acknowledges financial support from SUAM Foundation. 


\section{A Comptonization}

Consider a point at an optical depth $\tau_{e s}$ inside the medium. The mean number of scatterings of a soft photon escaping from this point is (Rybicki \& Lightman 1979)

$$
s \approx \tau_{e s}+\tau_{e s}^{2} .
$$

Let the average photon energy change per scattering be $A$, i.e. the initial and final photon energies, $E_{i n}$ and $E_{f i n}$, are related on average by $E_{f i n}=A E_{i n}$. For a thermal distribution of electrons with temperature $\theta=k T /\left(m_{e} c^{2}\right)$, we have (Rybicki \& Lightman 1979)

$$
A \approx 1+4 \theta+16 \theta^{2} .
$$

The Compton y-parameter is simply $y=s(A-1)=s\left(4 \theta+16 \theta^{2}\right)$.

After $j$ scatterings, the energy gain of the photon is $A^{j}$ so long as $E_{\text {fin }}$ is not saturated to the Wien regime $\left(E_{f i n} \sim 3 k T\right)$. The maximum $j$ corresponding to saturation is thus

$$
j_{m}=\ln \eta_{\max } / \ln A, \quad \text { where } \eta_{\max }=\eta_{W i e n}=\frac{3 k T}{E_{\text {in }}} .
$$

The energy enhancement factor is then simply the average energy gain of the photon:

$$
\eta=\sum_{j=0}^{j_{m}} A^{j} P_{j}+A^{j_{m}} \sum_{j=j_{m}+1}^{\infty} P_{j},
$$

where $P_{j}$ is the probability that a photon will suffer exactly $j$ scatterings.

To compute $\eta$, we need an expression for $P_{j}$. The probability that a photon travels a distance characterized by an optical depth $\tau_{e s}$ without scattering is $e^{-\tau_{e s}}$. To compute $P_{j}$, the assumption is usually made that in a medium with $\tau_{e s} \lesssim 1$, successive scattering events can be treated independently (e.g. Dermer, Liang, \& Canfield 1991), so that $P_{j}$ can be written as $e^{-\tau_{e s}}(1-$ $\left.e^{-\tau_{e s}}\right)^{j}$. However, for spherical geometry the scattering probability decreases after successive scatterings, because the mean square distance of a photon from the center increases with each scattering. Allowing for this accurately requires Monte Carlo calculations. One limit however, is straightforward, namely the case when the photon continues to travel radially outward after each scattering. In this case, the probability $P_{j}$ is given by the Poisson formula

$$
P_{j}=\frac{e^{-s} s^{j}}{j !}
$$

With the above expression for $P_{j}$ we can write $\eta$ as

$$
\begin{aligned}
\eta & =e^{-s}\left[\sum_{j=0}^{j_{m}} \frac{(A s)^{j}}{j !}+A^{j_{m}} \sum_{j=j_{m}+1}^{\infty} \frac{s^{j}}{j !}\right]= \\
& =e^{-s}\left[e^{A s}-\sum_{j=j_{m}+1}^{\infty} \frac{(A s)^{j}}{j !}+A^{j_{m}} \sum_{j=j_{m}+1}^{\infty} \frac{s^{j}}{j !}\right] .
\end{aligned}
$$

To evaluate the sums in equation $(30)$ we define the following function:

$$
f(x)=\sum_{j=j_{m}+1}^{\infty} \frac{x^{j}}{j !} .
$$


Using integration by parts and the fact that $f(0)=0$, it is easy to show that

$$
f(x) e^{-x}=\frac{1}{j_{m} !} \int_{0}^{x} e^{-y} y^{j_{m}} d y
$$

and therefore,

$$
f(x)=\sum_{j=j_{m}+1}^{\infty} \frac{x^{j}}{j !}=\frac{e^{x}}{j_{m} !} \int_{0}^{x} e^{-y} y^{j_{m}} d y=e^{x} P\left(j_{m}+1, x\right),
$$

where $P\left(j_{m}+1, x\right)$ is the incomplete gamma function. Substituting this result into equation (32) yields the final result:

$$
\begin{aligned}
\eta & =e^{-s}\left[\left(e^{A s}-e^{A s} P\left(j_{m}+1, A s\right)\right)+A^{j_{m}} e^{s} P\left(j_{m}+1, s\right)\right]= \\
& =e^{(A-1) s}\left[1-P\left(j_{m}+1, A s\right)\right]+\eta_{\max } P\left(j_{m}+1, s\right) .
\end{aligned}
$$




\section{References}

Abramowicz, M. A. 1996, in Basic Physics of Accretion Disks, ed. S. Kato, S. Inagaki, J. Fukue, \& S. Mineshige (Gordon \& Breach), in press

Abramowicz, M. A., Chen, X., Kato, S., Lasota, J. P., \& Regev, O. 1995, ApJ, 438, L37

Abramowicz, M. A., Czerny, B., Lasota, J. P., \& Szuszkiewicz, E. 1988, ApJ, 332, 646

Begelman, M. C. \& Chiueh, T. 1988,ApJ, 332, 872

Chen, X. 1995, MNRAS, 275, 641

Chen, X., Abramowicz, M. A., Lasota, J. P., Narayan, R., Yi, I. 1995, ApJ, 443, L61

Dermer, C. D., Liang, E. P., \& Canfield, E. 1991, ApJ, 369, 410

Frank, J., King, A., \& Raine, D. 1992, Accretion Power in Astrophysics (Cambridge, UK: Cambridge University press)

Gilfanov, M., et al. 1995, in The Lives of the Neutron Stars, NATO ASI Series, eds. M. A. Alpar, U. Kiziloglu \& J. van Paradijs (Kluwer), vol. 450, 331

Grebenev, S., et al. 1993, A\&AS, 97, 281

Harmon, B. A., et al. 1994, The Second Compton Symposium, eds. C. E. Fichtel, N. Gehrels \& J. P. Norris (New York: AIP), 210

Johnson,W. N., et al. 1994, The Second Compton Symposium, eds. C. E. Fichtel, N. Gehrels \& J. P. Norris (New York: AIP), 515

Kato, S., Abramowicz, M. A., \& Chen, X. 1995, PASJ, in press

Kinzer, R. L., et al. 1994, The Second Compton Symposium, eds. C. E. Fichtel, N. Gehrels \& J. P. Norris (New York: AIP), 531

Kusunose, M. 1996, in Basic Physics of Accretion Disks, eds. S. Kato, S. Inagaki, J. Fukue, \& S. Mineshige (Gordon \& Breach), in press

Kusunose, M. \& Takahara, F. 1985, Prog. Theor. Phys., 73, 41

Kusunose, M. \& Takahara, F. 1989, PASJ, 41, 263

Lasota, J. P., Abramowicz, M. A., Chen, X., Krolik, J., Narayan, R., \& Yi, I. 1996, ApJ, 462, 000

Lightman, A. P. \& Eardley, D. M. 1974, ApJ, 187, L1

Lynden-Bell, D. \& Pringle, J. E. 1974, MNRAS, 168, 603

Luo, C. \& Liang, E. P. 1994, MNRAS, 266, 386

Mahadevan, R, Narayan, R, Yi, I. 1996, ApJ, in press

Maisack, M., et al. 1993, ApJ, 407, L61

Melia, F. 1994, ApJ, 426, 577

Melia, F. \& Misra, R. 1993, ApJ, 411, 797

Narayan, R. 1996, ApJ, in press

Narayan, R., McClintock, J. E., \& Yi, I. 1996, ApJ, in press (Feb. 1, 1996)

Narayan, R. \& Popham, R. 1993, Nature, 362, 820

Narayan, R. \& Yi, I. 1994, ApJ, 428, L13

Narayan, R. \& Yi, I. 1995a, ApJ, 444, 231

Narayan, R. \& Yi, I. 1995b, ApJ, 452, 277

Narayan, R., Yi, I., \& Mahadevan, R. 1995, Nature, 374, 623

Narayan, R., Yi, I., \& Mahadevan, R. 1996, in Proc. Third Compton Symposium, A\&AS Special Issue, in press

Novikov, I. D. \& Thorne, K. S. 1973, in Blackholes ed. C. DeWitt \& B. DeWitt (New York: Gordon and Breach), 343

Pacholczyk, A. G. 1970, Radio Astrophysics (San Francisco: Freeman)

Phinney, E. S. 1981, in Plasma Astrophysics, eds. T. D. Guyenne \& G. Levy (ESA SP-161), 337 
Piran, T. 1978, ApJ, 221, 652

Pringle, J. E., Rees, M. J., \& Pacholczyk, A. G. 1973, A\&A, 29, 179

Rees, M. J., Begelman, M. C., Blandford, R. D., Phinney, E. S. 1982, Nature, 295, 17

Rybicki, G. B. \& Lightman, A. P. 1979, Radiative Processes in Astrophysics (New York: John Wiley \& Sons)

Shakura, N. I. \& Sunyaev, R. A. 1973, A\&A, 24, 337

Shapiro, S. L., Lightman, A. P., \& Eardley, D. M. 1976, ApJ, 204, 187

Svensson, R. 1982, ApJ, 258, 335

Svensson, R. 1984, MNRAS, 209, 175

Tanaka, Y. 1989, in Proc. 23rd ESLAB Symp. on Two Topics in X-Ray Astronomy, eds. J. Hunt \& B. Battrick (ESA SP-296), 3

Wandel, A. \& Liang, E. P. 1991, ApJ, 380, 84

White, T. R. \& Lightman, A. P. 1989, ApJ, 340, 1024 


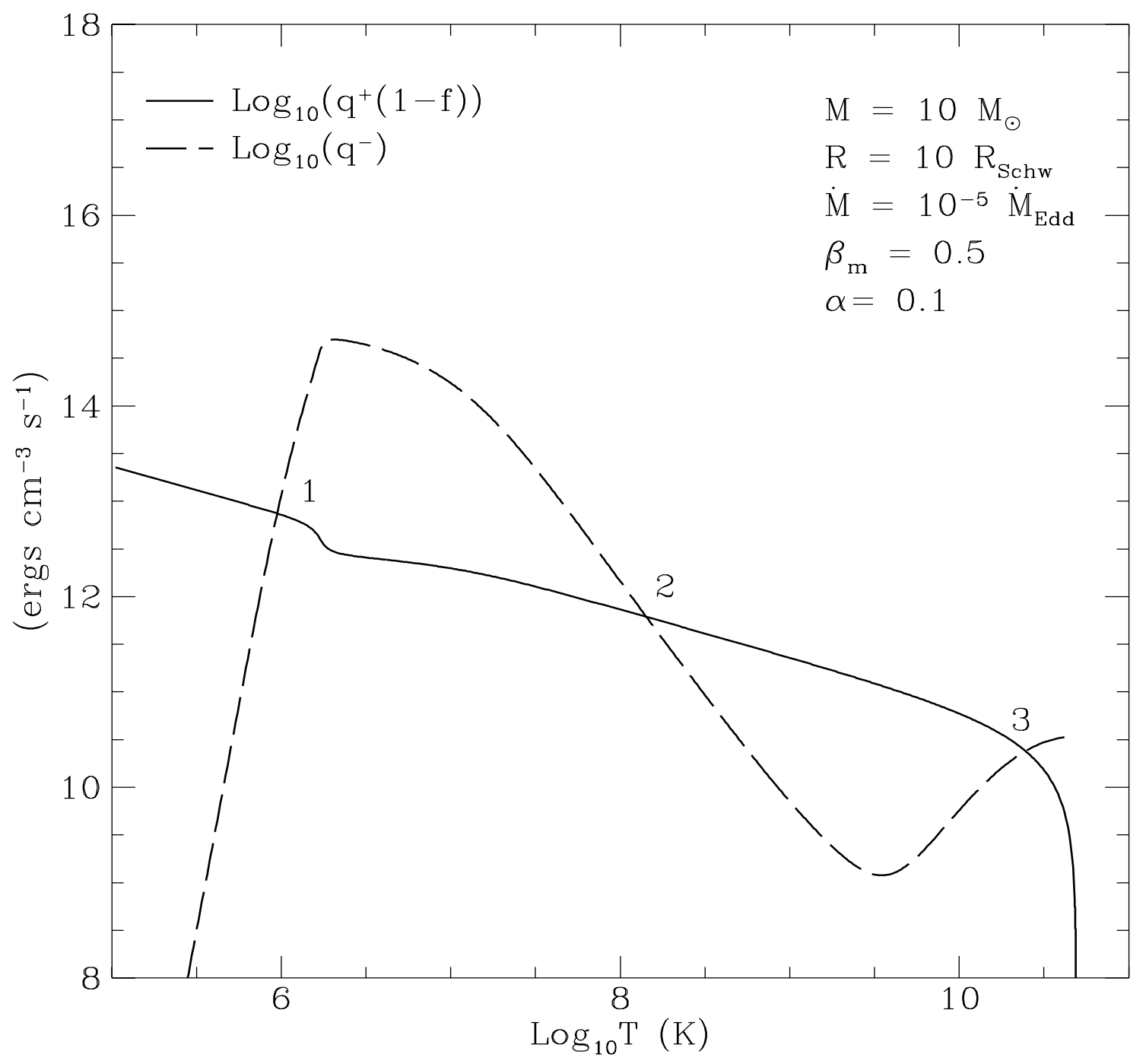

Figure 1: The rates of heating (solid line) and cooling (dashed line) of the accreting singletemperature gas plotted vs. gas temperature, $T$. Intersection points of the two curves mark equilibrium states of the system. Point 1 corresponds to the standard thin disk solution, point 2 is equivalent to the unstable SLE solution, and point 3 is our new, hot, thermally and viscously stable, solution. 

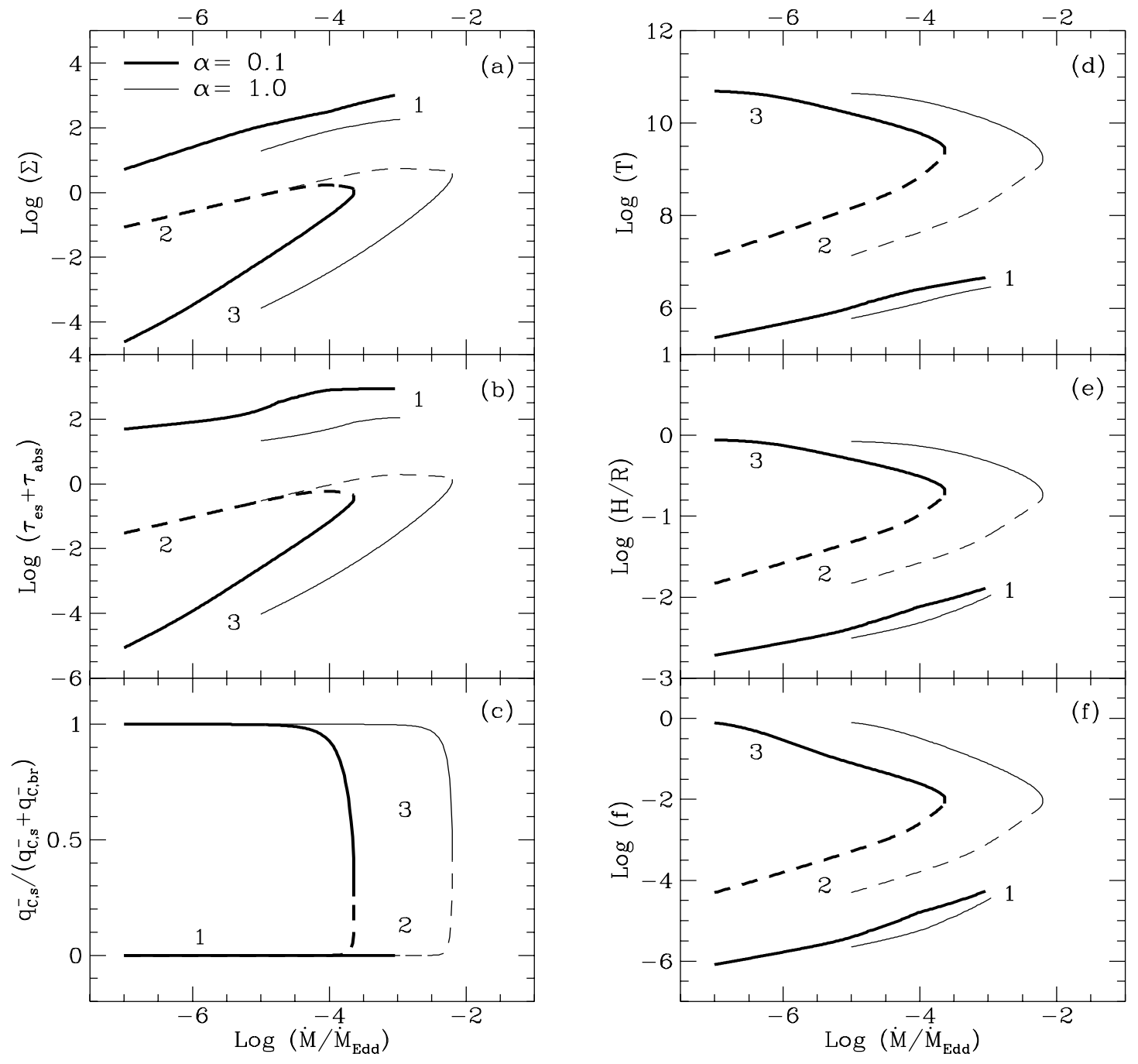

Figure 2: Thermal equilibria of single-temperature accretion disks with $\beta_{m}=0.5$ around a $10 M_{\odot}$ black hole at a radius of $R=10 R_{S c h w}$. The heavy and thin lines represent solutions with $\alpha=1$ and $\alpha=0.1$ respectively. The three branches labeled as 1,2 , and 3 correspond respectively to the standard thin disk, unstable SLE disk (indicated by a dashed line), and our hot and stable solution (as in Figure 1). In the six panels we plot (a) surface density, $\Sigma\left(\mathrm{g} \mathrm{cm}^{-2}\right)$, (b) total optical depth, $\left(\tau_{e s}+\tau_{a b s}\right)$, (c) fraction of the total cooling due to Comptonized synchrotron radiation, (d) temperature, $T(\mathrm{~K})$, (e) the vertical scale height, $H / R$, and (f) advected energy fraction, $f$, all as functions of the accretion rate $\dot{M}$. Note that the hot solution branch (labeled 3) is optically thin, has essentially spherical geometry $(H / R \approx 1)$, and cools mainly by synchrotron radiation. 

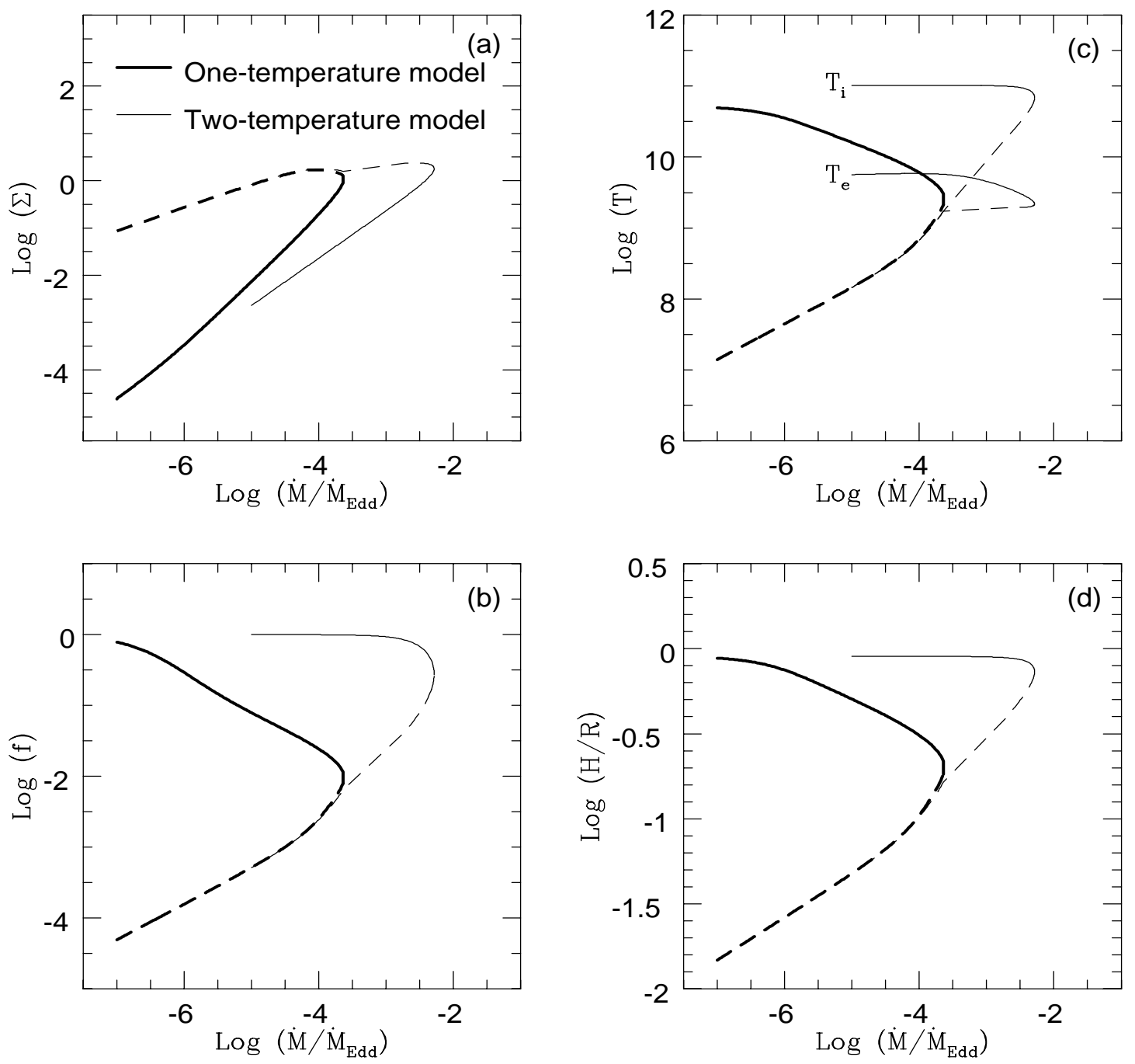

Figure 3: Thermal equilibria of single-temperature (heavy line) and two-temperature (thin line) accretion disks with $\beta_{m}=0.5$ and $\alpha=0.1$ around a $10 M_{\odot}$ black hole at a radius of $r=10$. The thin disk branch is not shown. The four panels show (a) surface density, $\Sigma\left(\mathrm{g} \mathrm{cm}^{-2}\right)$, (b) advection parameter, $f,(\mathrm{c})$ temperature, $T(\mathrm{~K})$, and $(\mathrm{d})$ the scale height, $H / R$, plotted as functions of $\dot{M}$. The unstable branch is shown by dashed lines. On the stable branch, the singletemperature solution is less advection-dominated and has a higher electron temperature than the two-temperature solution. 

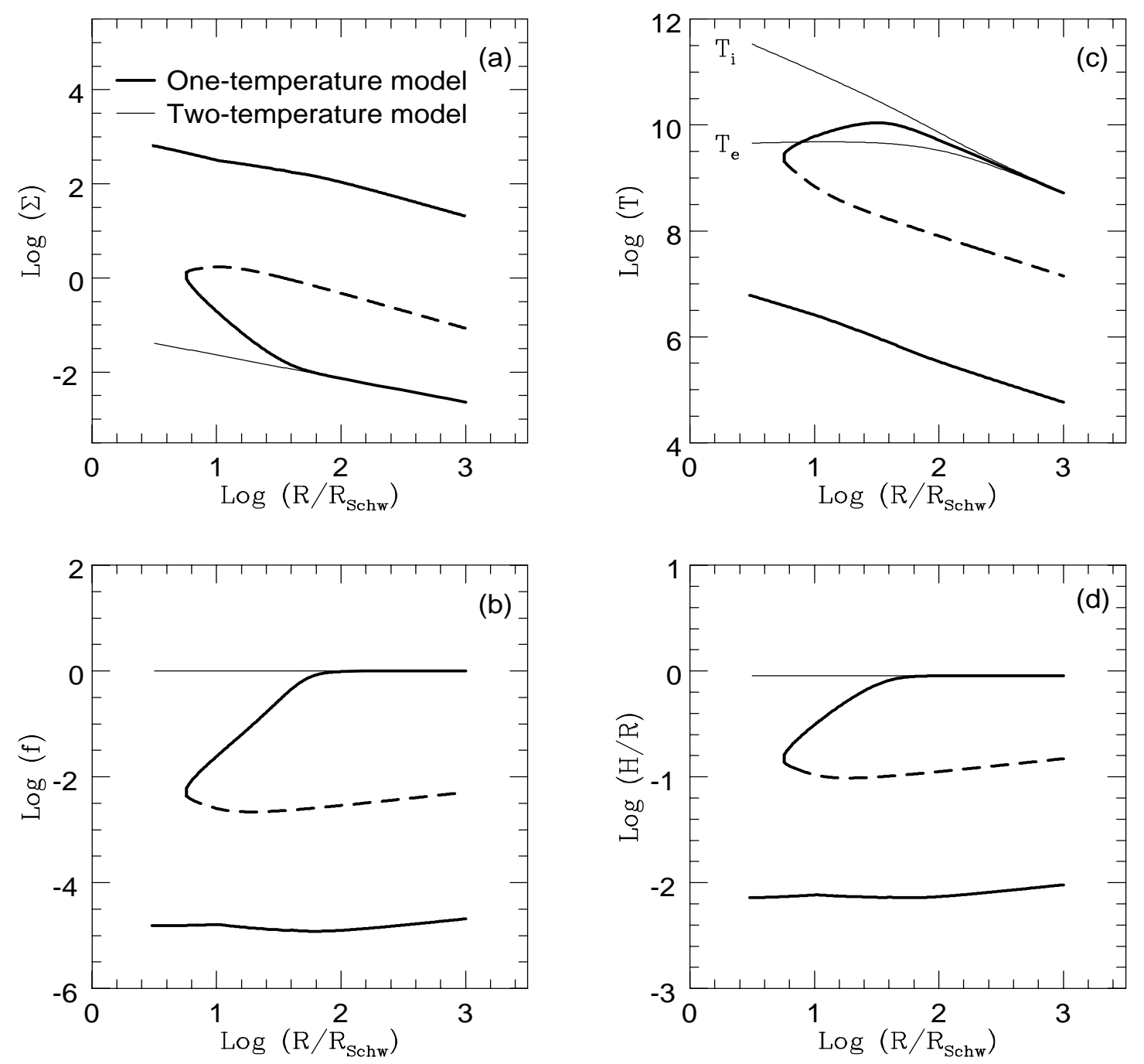

Figure 4: Radial profiles of thermal equilibria for a single-temperature accretion disk with $\beta_{m}=0.5, \alpha=0.1$, and $\dot{M} / \dot{M}_{E d d}=10^{-4}$ around a $10 M_{\odot}$ black hole (heavy line). For comparison we also plot the hot stable branch of the two-temperature disk (thin line). As expected, the two models give identical results at large radii. Closer to the black hole, however, the singletemperature solution becomes cooling-dominated while the two-temperature solution remains advection-dominated. Note the maximum in the disk temperature at $R \approx 40 R_{S c h w}$. 

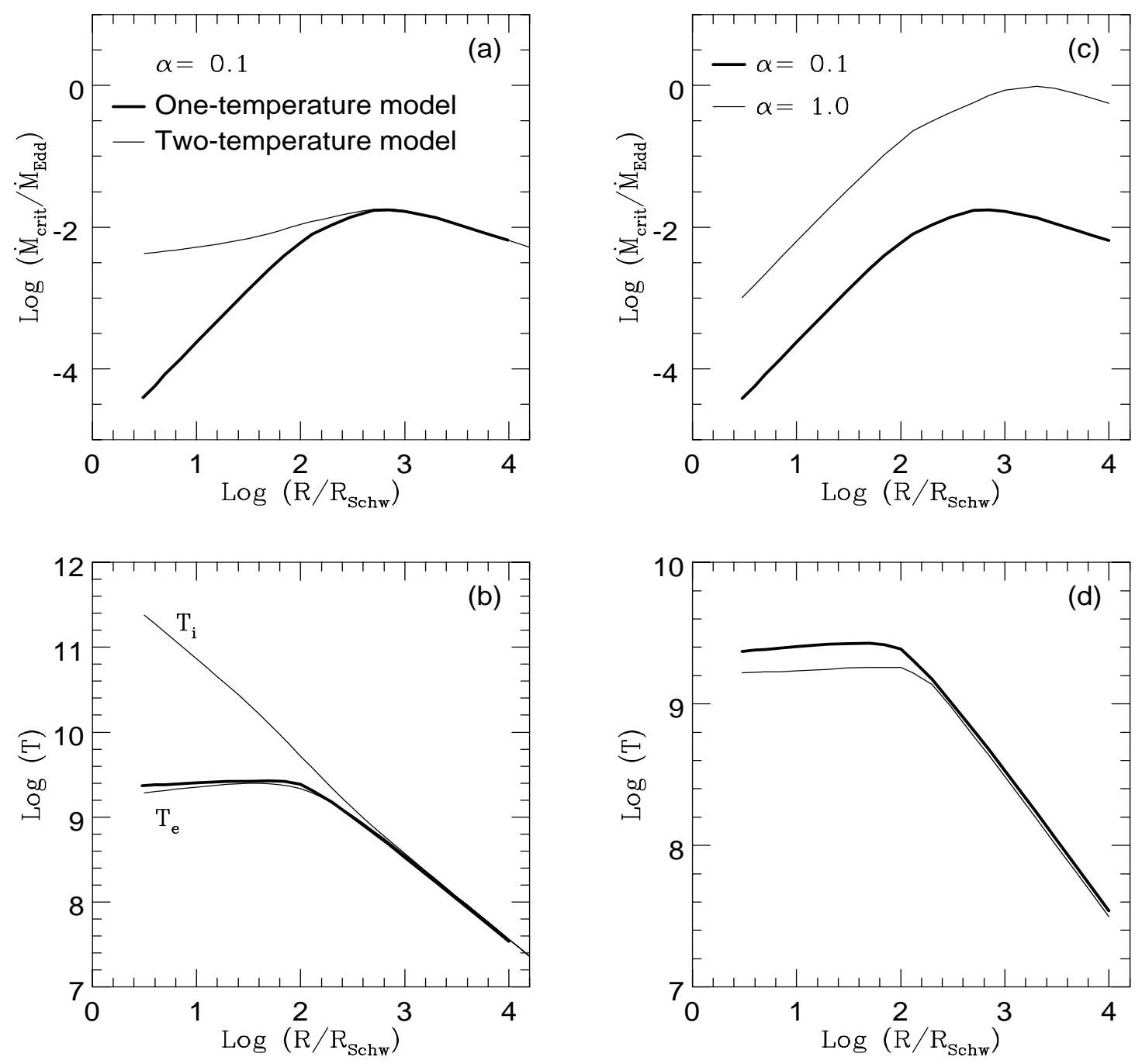

Figure 5: Critical accretion rate $\dot{M}_{\text {crit }}$ (upper panels) and corresponding temperature (lower panels) are plotted as functions of the radius. Panels (a) and (b) compare the results for the oneand two-temperature models for $\beta_{m}=0.5$ and $\alpha=0.1$. The critical $\dot{M}$ differs significantly in the two models, but the gas temperature in the single-temperature model is practically always equal to $T_{e}$ in the two-temperature model. Panels (c) and (d) show the results for a single-temperature disk with $\beta_{m}=0.5$ and two different values of $\alpha$. Note that although $\alpha=1.0$ allows models with values of $\dot{M}_{c r i t}$ higher by two orders of magnitude, the corresponding temperatures do not depend strongly on $\alpha$. 

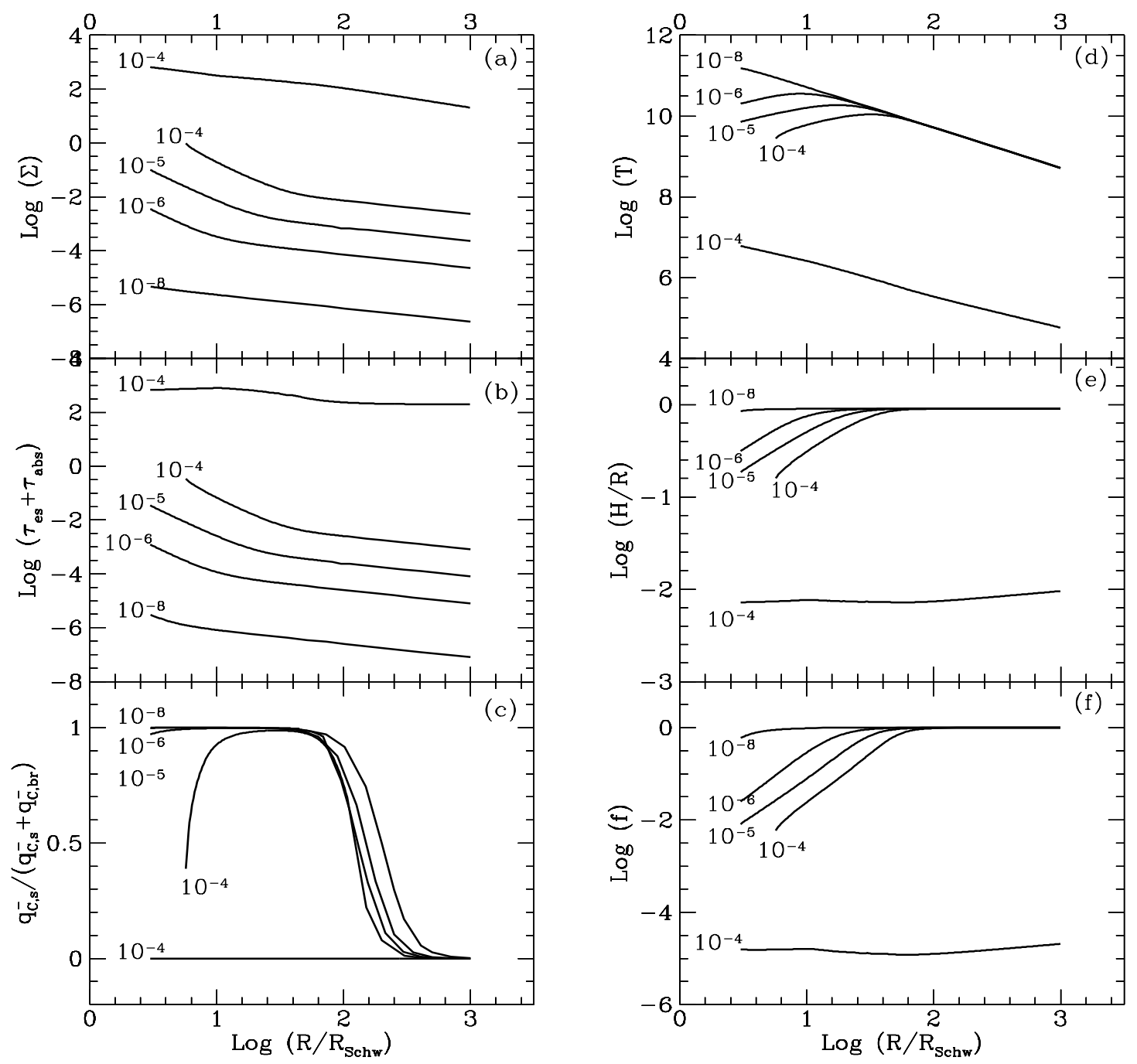

Figure 6: Radial profiles of hot single-temperature solutions, labeled by the values of the accretion rate in units of $\dot{M}_{E d d}$. The other parameters have the same values as in Figure 4 . Note that for $\dot{M}=10^{-4} \dot{M}_{E d d}$ the hot branch does not extend all the way to the inner edge of the disk at $R=3 R_{S c h w}$. Therefore, the only stable configuration at very small radii is the thin disk solution which is also shown on the figure. 

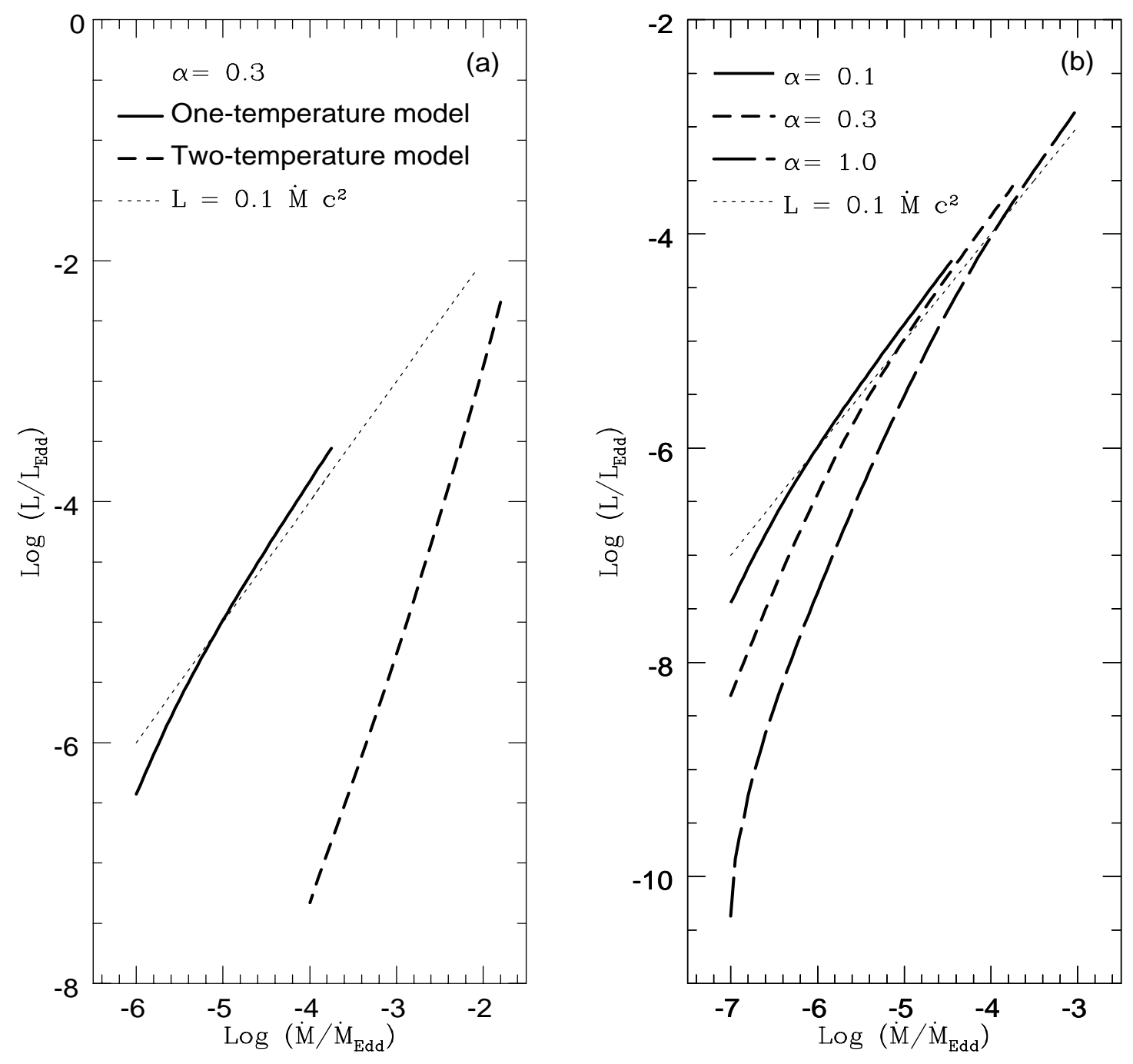

Figure 7: (a) Compares the integrated luminosities for one- and two-temperature hot accretion disks with $\beta_{m}=0.5, \alpha=0.3$, and $M=10 M_{\odot}$. The single-temperature disk is clearly more efficient than a two-temperature disk for a given $\dot{M}$ since its interior regions are coolingdominated. However, the limiting $\dot{M}_{c r i t}$ of the one-temperature solution is lower than that of the two-temperature flow by two orders of magnitude. (b) Integrated luminosity of a hot singletemperature accretion disk around a $10 M_{\odot}$ black hole as a function of the mass accretion rate, for $\beta_{m}=0.5$ and three different values of $\alpha$. Each curve was calculated up to the maximum value of the accretion rate given by $\dot{M}_{\text {max }}=\dot{M}_{\text {crit }}\left(R=3 R_{S c h w}\right)$. The dotted line shows the dependence expected if the radiative efficiency is $10 \%$. Note that disks with lower values of $\alpha$ are more luminous at a given $\dot{M}$, but have a lower limiting accretion rate $\dot{M}_{\text {crit }}$. 